\title{
PLAUSIBILIDADE E MÉTODO INDUTIVO NO USO DO PROJECT BASED LEARNING EM CURSOS DE ENGENHARIA
}

DOI: 10.37702/2175-957X.COBENGE.2021.3527

Guilherme Nami Bortolozi - nami.guilherme12@gmail.com

Instituto Mauá de Tecnologia

Rua Amadeu Vezzaro 130

09580-710 - São Caetano do Sul - SP

Octavio Mattasoglio Neto - omattasoglio@uol.com.br

Instituto Mauá de Tecnologia

Rua Tijuco Preto 933

03316-000 - São Paulo - SP

Resumo: O objetivo deste trabalho é identificar os elementos que permitem estabelecer uma relação entre a plausibilidade e os princípios do Project based Learning para professores de cursos de engenharia. A plausibilidade é uma das etapas para a aceitação de um determinado modelo de ensino e determina se os princípios desse modelo estão alinhados com as concepções que os professores têm sobre a aprendizagem dos estudantes. Sem esse alinhamento pode haver uma ação docente que não leve a uma efetiva prática dos princípios e etapas da estratégia de ensino, tornando-a menos efetiva para promover a aprendizagem. A plausibilidade emerge do modelo de mudança conceitual que tem a epistemologia e o construtivismo como referência. Outro elemento que será considerado como mediador da aprendizagem é o método indutivo, que considera que a experiência prática deve ser um dos pilares da aprendizagem em cursos de engenharia, pela característica dos objetos de aprendizagem dessa área de conhecimento. $O$ trabalho é puramente bibliográfico e tem como meta, identificar dentro dos referenciais adotados quais questões permitem identificar a plausibilidade do PBL, visando uma futura pesquisa com levantamento de dados junto a professores de cursos de engenharia. Como resultado, chegou-se a um conjunto de questões para essa pesquisa. $O$ trabalho constitui-se num ensaio relacionando três grandes elementos: o PBL, o modelo de mudança conceitual e a aprendizagem por indução.

Palavras-chave: Modelo de mudança conceitual. Project Based Learning. Plausibilidade. Formação de professores. Método indutivo. 


\section{PLAUSIBILIDADE E MÉTODO INDUTIVO NO USO DO PROJECT BASED LEARNING EM CURSOS DE ENGENHARIA}

\section{INTRODUÇÃO}

Qualquer novo conhecimento estabelecido deve ter a capacidade de resolver problemas gerados por seus antecessores. Caso contrário, esse conceito não será considerado como uma escolha aceitável. Para essa aceitação a plausibilidade é uma das condições sendo o resultado da consistência dos conceitos com outros conhecimentos da pessoa.

É com base nesses pressupostos que será discutido se as estratégias ativas são consistentes e plausíveis para os professores de cursos de Engenharia, ou seja, se são compatíveis com conhecimentos prévios dos docentes sobre a aprendizagem. Para isso este trabalho terá como base teórica o Modelo de Mudança Conceitual - MMC (POSNER et al, 1982), a estruturação do Project Based Learning (SILVEIRA et al, 2006) e um visão do modelo indutivo de aprendizagem (PRINCE e FELDER, 2006).

Este trabalho é essencialmente uma reflexão com base no entrelaçamento de ideias de três outros trabalhos, constituindo-se assim num ensaio sobre o ensino e a aprendizagem em engenharia. Nele se procura alinhar ideias, tendo como cenário o uso de estratégias ativas de aprendizagem e, mais precisamente o Project Based Learning - PBL. É, então, um exercício de promover a convergência das ideias desse grupo de autores, que marcam o ensino e a aprendizagem de conceitos científicos e o ensino e aprendizagem no campo Engenharia. O propósito é fundamentar a reflexão sobre a plausibilidade do uso do PBL por professores de Engenharia.

O MMC aponta que, seguido da etapa de insatisfação, a mudança para uma nova concepção passa pela etapa de inteligibilidade dos seus conceitos primordiais, sua estruturação e seus elementos mais complexos, seguida pela etapa da plausibilidade dos novos conceitos com outros conhecimentos prévios do sujeito, tais como: seus compromissos epistemológicos e suas crenças; conhecimentos e teorias advindos de suas formações; experiências vividas.

Em trabalhos anteriores, foram realizadas pesquisas sobre a satisfação dos professores quanto ao uso de estratégias ativas (SILVA e MATTASOGLIO Neto, 2019) e sobre a inteligibilidade no uso de estratégias ativa (BARBERINI e MATTASOGLIO Neto. 2020; MATTASOGLIO Neto, et al, 2020).

Neste novo trabalho, o foco está na etapa de plausibilidade que pode dar indícios tanto da adesão de professores a essa estratégia quanto à sua rejeição. Para buscar entender melhor a adesão dos professores ao Project Based Learning (PBL) o referencial teórico continua sendo o MMC de Posner et al. (1982), sendo que a opção por esse modelo teórico se deve ao paralelo traçado entre uma reestruturação de conceitos previamente concebidos na área de educação, com o processo de aprendizagem norteado para uma mudança do referencial pedagógico, podendo assim ser traçada uma relação análoga ao MMC.

O MMC tem como base a teoria de Thomas Khun sobre o desenvolvimento da ciência e a teoria de Piaget sobre a aprendizagem, que envolve a assimilação e acomodação como formas de apropriação do conhecimento (ARRUDA e VILLANI, 1994). Com base nesses alicerces teóricos, será conduzida uma investigação pautada nos princípios tangentes à ecologia conceitual dos referenciais adotados. E para a efetivação 
do método, o MMC dará suporte para a interpretação da aceitação ou não de um novo referencial teórico sobre aprendizagem.

Conhecer a aceitação dos professores de um novo referencial para promover o seu trabalho de ensino é importante porque uma fraca crença de sucesso numa estratégia pode levar o professor a não seguir seus pressupostos de modo adequado e ao sinal de dificuldades, voltar a agir dentro de um modelo anterior, por exemplo, o modelo tradicional de ensino ou, por outro lado, a não tirar todo o proveito do modelo que está praticando.

\section{FUNDAMENTAÇÃO TEÓRICA}

A fundamentação teórica desta pesquisa se apoia no MMC de Posner et al. (1982). Em seu artigo, Posner elabora parâmetros que definem um alinhamento com a mudança de preconcepções, que constituem o conhecimento intuitivo de senso comum e com o qual se interpreta o mundo, para novas ideias que são fundamentalmente melhor embasadas. A opção por esse modelo teórico se deve ao paralelo traçado entre uma reestruturação de conceitos previamente concebidos na área de educação, com o processo de aprendizagem norteado para uma mudança do referencial pedagógico, podendo assim ser traçada uma relação análoga ao MMC.

Em paralelo a isso, outro referencial usado está no artigo Inductive Teaching and Learning Methods: Definitions, Comparison, and Research Bases (PRINCE e FELDER, 2006) em que os autores estabelecem que e ensino de ciências e engenharia são tradicionalmente ensinadas dedutivamente, ou seja, supõem-se que o instrutor ou orientador irá apresentar um determinado tópico focando, primeiramente, sobre princípios gerais e, em seguida, usando tais princípios para derivar modelos matemáticos, mostrar aplicações ilustrativas dos modelos, para assim dar aos alunos a prática de derivações e aplicações semelhantes em trabalhos de casa e, finalmente, testar sua capacidade de fazer o mesmo em exames. Pouca ou nenhuma atenção é dada inicialmente à questão de por que tudo isso está sendo feito (PRINCE e FELDER, 2006), isto é, quais fenômenos do mundo real são explicados pelos modelos apresentados; quais problemas práticos podem ser resolvidos por esses métodos e por que os alunos devem se preocupar com isso. Com esses problemas em mente, o uso do MMC aplicado para projetos de Project Based Learning será o panorama principal para guiar a investigação bibliográfica deste trabalho. Sendo assim, vale convencionar as condições requisitadas para melhor análise do MMC, e também seu método de aplicação, que podem explicados da seguinte forma:

No MMC, o primeiro estágio da mudança ocorre a partir de uma anomalia, isto é, uma perceptível falha específica de uma determinada ideia, isso gera a iminente necessidade da elaboração de uma nova teoria moldada sobre um novo paradigma que irá substituir o anterior. A ideia básica do modelo é que a aprendizagem em ciência, que se dá com a passagem de concepções espontâneas para outras aceitas cientificamente, acontece quando algumas condições são satisfeitas: Insatisfação com as atuais concepções e o contato com novas concepções que sejam inteligíveis, plausíveis e férteis. (MATTASOGLIO Neto e PAVÃO, 2006).

Tais condições são parâmetros subsequentes aos quais devem ser vistas como conceitos centrais de uma pessoa que irão construir território para a fundamentação de novas ideias. Porém vale ressaltar que esse tipo de consideração sugere que há várias condições importantes que devem ser atendidas antes que uma acomodação ocorrer. Para 
este trabalho, o enfoque vai estar na terceira etapa de análise do MMC, que pode ser definida como:

- Plausibilidade - Em uma terceira etapa do processo, a análise estará nos fatores de mostrar como sendo plausível a aplicação da metodologia referida, para isso será medido se a nova concepção é compatível com outros conhecimentos do sujeito, como: crenças e compromissos epistemológicos; outras teorias ou conhecimentos; experiências anteriores. Deve-se, ainda, resolver problemas conhecidos e as anomalias que desencadearam a busca de novas concepções e criar imagens para os conceitos sobre o que é ou pode ser o mundo (MATTASOGLIO Neto e PAVÃO, 2006). Os valores relacionados ao paradigma tradicional, podem levar a reações contra as mudanças. Isto indica a necessidade de uma ação sistemática de abertura à fala dos participantes para que retrocessos não comprometam o processo de mudança (POSNER, 1982).

Outro ponto chave do MMC está na interpretação da ecologia conceitual do sujeito, isto é, seus conceitos atuais que irão influenciar a seleção de um novo conceito central. Os seguintes tipos de conceitos são determinantes na direção de uma nova acomodação: às próprias anomalias; analogias e metáforas, utilizadas para a sugestão e inteligibilidade das novas ideias; compromissos epistemológicos do sujeito, relacionados com os ideais explicativos e pontos de vista gerais acerca do conhecimento, que exigem atributos relevantes como: elegância; economia; parcimônia do conceito ou teoria; crenças e conceitos metafísicos sobre os conceitos específicos e sobre a própria ciência; e outros conhecimentos, de outros campos ou conceitos competitivos (ARRUDA e VILLANI, 1994).

A plausibilidade, juntamente com as etapas precedentes, insatisfação e inteligibilidade, pode agir como interferências ou catalisadores no processo de interpretação de novos conhecimentos. O MMC considera também a existência de preconcepções que constituem o conhecimento intuitivo de senso comum e com o qual se interpreta o mundo, para novas ideias que são fundamentalmente melhor embasadas. Estas concepções prévias são parcialmente consistentes e estáveis, resistindo à mudança (MATTASOGLIO Neto e PAVÂO, 2006). Os conceitos interpretados para validação de mudança são aqueles referentes ao processo de ensino-aprendizagem, que será tratada paralelamente ao conceito de mudança de paradigma de Posner. Tais conceitos estão diretamente relacionados à aceitação de um modelo construtivista de aprendizagem em contraposição ao fazer tradicional, tal como indicado por Mizukami (1992). E este modelo será amplamente utilizado para estimar e interpretar vias do método de mudança paradigmática no processo de ensino-aprendizagem.

Em termos referentes a plausibilidade do processo, o MMC afirma que "Uma nova concepção deve parecer inicialmente plausível". Qualquer novo conceito adotado deve pelo menos parecer ter a capacidade de resolver os problemas gerados por seus antecessores. Caso contrário, não parecerá uma plausível escolha. A plausibilidade também é resultado da consistência dos conceitos com outro conhecimento. Uma nova ideia em, digamos, astronomia é menos provável de ser aceito se for inconsistente com o conhecimento físico atual ou se simplesmente não tem uma conta física clara. Cientistas físicos antes do século 20, por exemplo, estavam relutantes em aceitar o que as genealogias afirmavam sobre a idade do mundo, uma 
vez que não tinham teoria que permitisse a soma para fornecer energia para esse período de tempo (POSNER et al. 1982).

Para contextualizar os parâmetros e referentes vistos na fundamentação teórica iremos assumir a mudança de conceito como sendo relacionada à mudança do referencial pedagógico para o processo de ensino-aprendizagem. Ou seja, os conceitos abordados na investigação devem ser interpretados como relativos aos modelos de aprendizagem, e por mudança de conceito entende-se como vias de abordagem aos paradigmas estabelecidos no processo de aprendizagem na área de engenharia.

O Project Base Learning - PBL - tem uma estruturação que vai ao encontro do modo de trabalho executado pelo Engenheiro, dessa forma é uma estratégia de ensino que tem uma adaptação muito interessante para o ensino de Engenharia. No trabalho de Sesoko e Mattasoglio Neto (2014) foi apresentada uma análise e uma estruturação do uso do PBL, seja na versão Project como na versão Problem, nos ensinos de Engenharia. O trabalho apresenta um paralelo entre essas duas modalidades, Project e Problem, e uma lista de dificuldades no uso dessas estratégias.

Silveira et al (2006), colocam as etapas do PBL e forma sintética pode ser descrita como:

$$
\begin{gathered}
\text { observação } \rightarrow \text { modelagem } \rightarrow \text { concepção } \rightarrow \text { otimização } \rightarrow \text { simulação } \rightarrow \text { implementação } \\
\text { ou implantação } \rightarrow \text { validação } \rightarrow \text { comunicação. }
\end{gathered}
$$

É nesse formato do PBL que ser irá analisar o MMC e o método de ensino e aprendizagem indutivo.

\section{METODOLOGIA}

Este trabalho tem como método a revisão bibliográficas em artigos pautados em metodologias de ensino-aprendizagem ativa, isto é, a condução de uma pesquisa bibliográfica como prospecção de pesquisas sobre concepções de aprendizagem, sobre o MMC e sobre a formação de professores.

Vale mencionar o cuidado estabelecido na condução da investigação, sendo que aquilo que será medido tem por objetivo estabelecer parâmetros de plausibilidade nas iniciativas estudadas. O filtro de pesquisa está na contextualização de metodologias de ensino, mais precisamente do PBL, e como a sua aplicação se relaciona aos princípios do $M M C$ referenciado na fundamentação teórica.

A má qualidade da revisão da literatura compromete todo o estudo, uma vez que esta não se constitui em uma seção isolada, mas, ao contrário, tem por objetivo iluminar o caminho a ser trilhado pelo pesquisador, desde a definição do problema do problema até a interpretação dos resultados. Para isto, ela deve servir a dois aspectos básicos: 1) a contextualização do problema dentro da área de estudo; e 2) a análise do referencial teórico. (ALVES, 1992).

Portanto, a revisão da literatura deste trabalho irá se apoiar em analisar como a visão dos professores e suas metodologias de ensino podem ir ao encontro com paradigmas préestabelecidos em sistemas de ensino-aprendizagem vigente. Para tanto, tal investigação deve medir se a nova concepção é compatível com outros conhecimentos e visões de mundo do sujeito, como já mencionado antes: crenças e compromissos epistemológicos; 
outras teorias ou conhecimentos; experiências anteriores. Essa medição deve se adaptar aos paradigmas de ensino no curso de engenharia, e abordar qual a visão que cada professor tem sobre metodologias de ensino que se baseiam no Project Based Learning, além disso, uma parte importante da investigação está em medir se o professor vê sentido em aplicar esses métodos em suas aulas, ou seja, se os princípios que serão reestruturados pelo MMC são compatíveis com a forma que o professor conduz ou pretende conduzir suas práticas de ensino.

É possível também que o professor entenda a aplicação do PBL mas não concorde em adotar suas novas medidas. Com isso em mente, cabe a investigação estimar quais foram os parâmetros que não estão de acordo com a visão de cada docente. É possível perceber que terão muitas variáveis a serem analisadas na inquisição de novos parâmetros, portanto o MMC deve ter cuidado em estabelecer mudanças que não saiam de um padrão visto pela opinião majoritária dos docentes daquilo que deve ou não ser reestruturado.

O apoio na fundamentação teórica é imprescindível na conduta da investigação, principalmente pelo fato de que o MMC deve considerar a existência de preconcepções que constituem o conhecimento intuitivo de senso comum e com o qual os professores interpretam suas próprias metodologias de ensino, e com isso em mente elaborar novas parâmetros que possivelmente sejam mais efetivos e práticos na visão desses professores.

\section{RESULTADOS: AS PERGUNTAS INICIAIS}

Define-se plausibilidade como sendo a medida daquilo que é plausível, isto é, aceitável ou admissível. Tal definição pressupõe que essa medição será feita através do estudo de parâmetros que confirmem ou não o grau de aceitação do objeto de estudo. É possível que algo seja coerente, mas não seja plausível, ou seja, o indivíduo entende os argumentos estabelecidos, mas não concorda com sua aplicação no contexto referido, interpretando suas medidas como destoantes de paradigmas próprios do sujeito.

No contexto deste trabalho, ser plausível está na medida em que o professor entende que a forma como o aluno aprende, vai ao encontro da forma como as metodologias ativas e também o PBL criam construções cognitivas que auxiliam o processo de aprendizagem do aluno. Partindo disso é possível estabelecer um paralelo com relações de aprendizagem que partem de um viés construtivista, que podem ser definidos como modelos que argumentam que, havendo ou não uma realidade objetiva, os indivíduos ativamente constroem e reconstroem sua própria realidade em um esforço para dar sentido à sua experiência.

As novas informações são filtradas por meio de estruturas mentais que incorporam o conhecimento prévio, crenças, preconceitos e equívocos, preconceitos e medos do aluno. Se a nova informação for consistente com essas estruturas, ela pode ser integrada a elas, mas se for contraditória, pode ser memorizada para o exame, mas é improvável que seja realmente incorporada ao sistema de crenças do indivíduo. (PRINCE e FELDER, 2006).

A convergência das estratégias ativas de aprendizagem e nelas considerando o PBL, com a percepção de que o aluno é o protagonista do processo de aprendizagem pela interação com o objeto de aprendizagem e que o processo indutivo permite aproximação do estudante com esse processo, de modo a superar até mesmo preconceitos e equívocos na interpretação dos fenômenos, a plausibilidade num processo de ensino desta natureza, está em o professor ter plena convicção que deve efetivamente criar situações que 
coloquem o aluno em contato com aquilo que aprende. Neste sentido o PBL apresenta etapas que permite essa interação e vai além, permitindo a interação interpessoal entre os aprendizes e entre esses e o professor.

\subsection{Como medir a plausibilidade?}

Apoiado nos princípios mencionados anteriormente, o MMC deve enfatizar que os princípios de aprendizagem nas estratégias ativas e, no PBL devem ser estruturados de forma a convergir a ecologia conceitual do aluno com a visão do corpo docente do que são metodologias de ensino eficientes. Nesse processo irão haver (ou poderá haver) alguns entraves a serem debatidos, como por exemplo o senso daquilo que é contraintuitivo, tanto para um aluno não familiarizado com determinado conceito ou paradigma, quanto para um professor que interpreta que o alinhamento às novas concepções de ensino e suas estratégias não são fundamentais para a eficiência nas mudanças desejadas.

Sendo assim a investigação deve priorizar em estabelecer como sendo plausível a aplicação da metodologia referida, para isso será medido se a nova concepção é compatível com outros conhecimentos dos professores sobre aprendizagem. E voltando ao que foi apresentado na Fundamentação Teórica, deve-se também, resolver problemas conhecidos e as anomalias que desencadearam a busca de novas concepções e criar imagens para os conceitos sobre o que é ou pode ser o mundo. (MATTASOGLIO Neto e PAVÃO, 2006).

Nesse sentido, as questões que se colocam devem ir ao encontro da estrutura de PBL apontada no trabalho de Silveira (2008), que são:

- Fornecer/escolher o tema do projeto.

- Coletar fatos: entender o projeto proposto (pesquisa inicial), formular os problemas envolvidos e estabelecer os objetivos do trabalho.

- Criar ideias para resolver ou elaborar o projeto.

- Aprender os conteúdos necessários para a realização do projeto/produto.

- Discussão das propostas de solução e realização do trabalho, sua viabilidade e chega-se a uma solução a ser implantada.

- Elaboração e implementação do projeto/produto.

- Realização de testes, coletando dados e verificando os resultados obtidos.

- Elaboração de um relatório escrito e de um seminário (apresentação oral) contendo: o objetivo, a descrição do projeto, a metodologia aplicada, os resultados e análises realizadas e, por fim, a conclusão do grupo com relação ao trabalho.

A reflexão sobre o tema leva a alguns questionamentos a serem respondidos:

- O professor percebe que o aluno traz concepções prévias sobre o projeto que irá realizar?

- O estudante tem a possibilidade de testar suas concepções prévias como solução para os problemas propostos?

- As soluções prévias estão alinhadas com o conhecimento em engenharia ou são de senso comum que não atendem na solução de problemas?

- O método indutivo se contrapõe ou complementa o dedutivo?

- Em que medida a estrutura do PBL está alinhada com o método indutivo?

São essas as perguntas inicias que devem conduzir o trabalho de investigação das concepções dos professores e a plausibilidade das concepções associadas ao PBL, sem o que não há garantia de que o PBL seja efetivamente incorporado e prático de fato. 


\section{CONSIDERAÇÕES FINAIS}

O enfoque principal deste trabalho é identificar se as concepções das estratégias de aprendizagem ativa são plausíveis para determinado corpo docente. Para isso o alinhamento entre o MMC e as ideias de Felder e Prince, dentro do referencial do PBL é o ponto de convergência do trabalho. Esse resultado permitirá identificar e avaliar as restrições e dificuldades na implementação de estratégias ativas para aprendizagem, mais precisamente do PBL. Como consequência, o resultado da pesquisa deverá abrir caminho para a indicação de formas de melhorar o desempenho no uso de estratégias ativas para aprendizagem, pela criação de mecanismo que ajudem na superação do desalinhamento entre as concepções dessas estratégias e aquelas dos professores sobre a construção do conhecimento, ou seja a aprendizagem.

$\mathrm{O}$ alinhamento dos professores às novas concepções de ensino e suas estratégias é fundamental para a eficiência nas mudanças desejadas. Nesse sentido, a identificação de pontos que dificultam a adesão dos professores às novas estratégias é algo que deve ser alcançado. De modo geral, os resultados permitirão uma avaliação da compreensão das estratégias ativas e a indicação de ações que podem promover a mudança conceitual de professores, visando uma adesão ao uso das estratégias ativas para a aprendizagem.

\section{Agradecimentos}

Ao IMT pelo apoio à iniciação científica do estudante dentro do projeto.

\section{REFERÊNCIAS}

ALVES, A.J., A "Revisão da Bibliografia" em teses e dissertações: meus tipos inesquecíveis. Cad. Pesq. São Paulo, n.81, p.53-60, maio 1992.

ARRUDA, S. M., VILLANI, A. Mudança Conceitual no Ensino de Ciências. Caderno Catarinense de Ensino de Física. Florianópolis: Editora da UFSC, v. 11, n. 2, p. 88$99,1994$.

FELDER, R.M., PRINCE, M., Inductive Teaching and Learning Methods: Definitions, Comparisons, and Research Bases. J. Engr. Education, 95(2), 123-138 (2006).

FELDER, R.M., BRENT, R., How to Evaluate Teaching. Chemical Engineering Education, 38(3), 200-202 (2004).

HEWSON, P. W. Conceptual change in science teaching and teacher education. National Center for Educational Research, Documentation, and Assessment, Madrid, Spain. 1992.

MATTASOGLIO Neto, O., PAVÃO, A. C., Estudo da implementação de uma proposta contextualizadora e ativa nas aulas do Ciclo Básico de um curso de Engenharia - A construção do Projeto Pedagógico. Anais: Congresso Brasileiro de Ensino de Engenharia. Passo Fundo, RS. 2006.

NADELSON, L. S.; HEDDY, B. C; JONES, S.; TAASOOBSHIRAZI, G. \& JOHNSON, M. Conceptual Change in Science Teaching and Learning: Introducing the Dynamic Model of 
Conceptual Change. International Journal of Educational Psychology, 7(2), 151-195, 2018.

POSNER, G. T. et al. Accommodation of a Scientific Conception: Towards a Theory of Conceptual Change. Science Education. v. 66, p. 211-227, 1982.

SILVEIRA, M. A. et al. Projeto LAPIN: um caminho para a implementação do aprendizado baseado em projetos. Anais: XXXVI - Congresso Brasileiro de Ensino de Engenharia. São Paulo: ABENGE, 2008.

TREINTA, F. T. et al. Metodologia de pesquisa bibliográfica com a utilização de método multicritério de apoio à decisão. Production, v. 24, n. 3, p. 508-520, July/Sept. 2014

\title{
THE PLAUSIBILITY OF ACTIVE STRATEGIES IN THE PERCEPTION OF ENGINEERING COURSE TEACHERS
}

\begin{abstract}
The objective of this work is to identify the elements that allow establishing a relationship between plausibility and the principles of Project based Learning from teachers of engineering courses. Plausibility is one of the steps for the acceptance of a given teaching model and determines whether the principles of that model are in line with the conceptions that teachers have about student learning. Without this alignment, there can be a teaching action that does not lead to an effective practice of the principles and stages of the teaching strategy, making it less effective to promote learning. Plausibility emerges from the conceptual change model that has epistemology and constructivism as a reference. Another element that will be considered as a learning mediator is the inductive method, which considers that practical experience must be one of the pillars of learning in engineering courses, due to the characteristic of the learning objects in this area of knowledge. The work is purely bibliographic and aims to identify, within the adopted references, which issues allow to identify the plausibility of the PBL, aiming a future research with data collection with professors of engineering courses. As a result, a set of questions was reached for this research. The work consists of an essay relating three major elements: the PBL, the conceptual change model and induction learning.
\end{abstract}

Keywords: Conceptual change model. Project Based Learning. Plausibility. Teacher training. Inductive method. 